\title{
Correlation analysis between liver metastasis and serum levels of miR-200 and miR-141 in patients with colorectal cancer
}

\author{
MENG DING ${ }^{1}$, TAO ZHANG ${ }^{2},{\text { SHIJIE } \text { LI }^{3}, \text { YING ZHANG }^{1}, \text { YUNFENG QIU }^{1} \text { and BIN ZHANG }}^{1}$ \\ ${ }^{1}$ Endoscopy Center; ${ }^{2}$ Department of Gastrointestinal Colorectal and Anal Surgery; ${ }^{3}$ Department of Thyroid Surgery, \\ China-Japan Union Hospital of Jilin University, Changchun, Jilin 130000, P.R. China
}

Received August 4, 2016; Accepted September 6, 2017

DOI: $10.3892 / \mathrm{mmr} .2017 .7538$

\begin{abstract}
Colorectal cancer has become a disease with one of the highest incidence rates worldwide, and liver metastasis threatens the prognosis of colorectal cancer. MicroRNA (miR)-200 and miR-141 have been confirmed to be aberrantly expressed in colorectal cancer, however, whether miR-200 and miR-141 are involved in the progression of colorectal cancer, and their role in liver metastasis remain to be fully elucidated. The aim of the present study was to investigate the role of miR-200 and miR-141 in the progression and liver metastasis of colorectal cancer in patients. A total of 380 patients with colorectal cancer were enrolled, of which 142 were diagnosed with grade I/II disease and 238 were grade III/IV disease. Reverse transcription-quantitative polymerase chain reaction analysis was performed to examine the serum levels of miR-200 and miR-141. In addition, transfection was performed in HCT116 and SW480 cells to establish overexpression models for miR-200 and miR-141. A TUNEL assay was performed to assess apoptosis and a Transwell experiment was performed to examine cell migration. Compared with the control group, the serum levels of miR-200 and miR-141 were significantly increased in the colorectal cancer group $(\mathrm{P}<0.05)$. Compared with the patients with colorectal cancer without liver metastasis, patients with liver metastasis had significantly higher serum levels of miR-200 and miR-141 $(\mathrm{P}<0.05)$. The overexpression of miR-200 and miR-141 inhibited apoptosis of the HC116 and SW480 cells, and enhanced cell migration. The upregulation of serum miR-200 and miR-141 were associated with liver metastasis in patients with colorectal cancer. Taken together, the overexpression of miR-200 and miR-141 exacerbated liver
\end{abstract}

Correspondence to: Dr Bin Zhang, Endoscopy Center, China-Japan Union Hospital of Jilin University, 126 Xiantai Street, Changchun, Jilin 130000, P.R. China

E-mail: binzhangzxc@163.com

Key words: liver metastasis, colorectal cancer, microRNA-200, microRNA-141, apoptosis, migration metastasis of colorectal cancer via inhibiting apoptosis and inducing migration.

\section{Introduction}

Colorectal cancer has become one of the malignant tumor diseases with the highest incidence rates worldwide. The liver is the main target organ for the hematogenous metastasis of colorectal cancer, and liver metastasis is the leading cause of colorectal cancer-associated mortality in patients (1). Clinical trials have shown that liver metastasis is an independent predictor of poor prognosis and increased risk of mortality in patients with colorectal cancer (2). There remains no effective therapy for liver metastasis despite promising developments achieved in the treatment of colorectal cancer. Early diagnosis and positive therapy may improve the survival rates of patients with colorectal cancer at an early stage of liver metastasis. Therefore, the present study aimed to examine the molecular mechanisms of liver metastasis in colorectal cancer, and screen for biomarkers associated with liver metastasis. These investigations are likely to assist in improving the accuracy of early diagnosis, efficacy of novel therapy and prognosis of patients with colorectal cancer (3).

MicroRNAs are a type of non-coding RNA with a length of 21-23 bp (4). Previous studies have shown that microRNAs affect multiple cellular events via mRNA interference (5). The microRNA expression profile of colorectal cancer shows differential expression of microRNAs in colorectal cancer, compared with normal tissues, suggesting that the aberrant expression of microRNA is associated with the metabolism and cell behavior of cancer (6). Several microRNAs have been confirmed to be associated with the clinicopathological features of colorectal cancer, including microRNA (miR)-31, miR-143 and miR-145 (7,8). Zhang et al (9) found that miR-200 was important in the regulatory mechanisms of liver cancer, and a previous study showed that serum levels of miR-200 and miR-141 were increased in patients with colorectal cancer (10). These findings suggest that miR-200 and miR-141 may be involved in the pathogenesis of colorectal cancer. However, whether the aberrant expression of miR-200 and miR-141 is associated with liver metastasis remains to be elucidated. The present study aimed to examine the association between liver metastasis and levels of serum miR-200 and miR-141 in patients with colorectal cancer. 


\section{Patients and methods}

Study subjects. A total of 380 patients with colorectal cancer were randomly enrolled into the experimental group at China-Japan Union Hospital of Jilin University (Changchun, China) between June 2008 and June 2014 (male/female ratio, 218/162; age range, $42-73 ; 53.6 \pm 6.2$ years). The patients with colorectal cancer were diagnosed by an alimentary tract barium meal, B-ultrasonic examination and histopathological analyses. Disease grading was performed for all enrolled patients according to the criteria of the World Health Organization grading standard for colorectal cancer (11). The grading comprised 142 grade I/II cases and 238 III/IV cases. None of the patients had received radiotherapy, chemotherapy or surgery prior to hospitalization. A control group included healthy adults, who were age- and sex-matched to patients in the experimental group. This clinical trial was performed following approval and under supervision of the Ethics Committee of the China-Japan Union Hospital of Jilin University (Changchun, China). All participants signed informed consent prior to enrollment in the study.

Reagents and instruments. RNA extraction kits (cat. no 74104) were purchased from Qiagen, Inc. (Valencia, CA, USA). Routine kits for reverse transcription-quantitative polymerase chain reaction (RT-qPCR) analysis (cat. no. KR103-03) were purchased from TianGen Biotech Co., Ltd. (Beijing, China). MicroRNA qRT-PCR kits (cat. no. AM1558) were purchased from Ambion; Thermo Fisher Scientific, Inc. (Waltham, MA, USA). Lipidosome INTERFERin ${ }^{\mathrm{TM}}$ transfection kits (cat. no. 410-015) were purchased from Polyplus Transfection (Illkirch, France). DMEM (cat. no. SH30081.01) was purchased from HyClone; GE Healthcare Life Sciences (Logan, UT, USA). TUNEL apoptosis detection kits were purchased from Roche Diagnostics (Basel, Switzerland). Transwell kits were purchased from BD Biosciences (Franklin Lakes, NJ, USA). The Mx3000P fluorescence ration PCR instrument was purchased from Agilent Technologies, Inc. (Santa Clara, CA, USA). The cell incubator was purchased from Thermo Fisher Scientific, Inc.

Extraction of microRNA and RT-qPCR analysis. Fasting blood samples $(10 \mathrm{ml})$ were collected into vacuum tubes with EDTA as an anticoagulant. Plasma was obtained via two-step centrifugation $(2,000 \mathrm{~g}$ for $10 \mathrm{~min}$; 8,000 $\mathrm{g}$ for $10 \mathrm{~min}$ at $25^{\circ} \mathrm{C}$ ). The supernatant was recycled for the extraction of total RNA and microRNA, respectively. Primers were synthesized for miR-200 and miR-141 (Table I). RT-qPCR analysis was performed in a total volume of $20 \mu \mathrm{l}$, including $10 \mu \mathrm{l}$ SYBR-Green qPCR Super mix, $0.5 \mu 1$ forward primer $(10 \mu \mathrm{M}), 0.5 \mu \mathrm{l}$ reverse primer $(10 \mu \mathrm{M}), 5 \mu \mathrm{l} \mathrm{cDNA}$ and $4 \mu \mathrm{l}$ sterile water using routine protocols. The reaction conditions were as follows: $95^{\circ} \mathrm{C}$ for $3 \mathrm{~min}, 95^{\circ} \mathrm{C}$ for $15 \mathrm{sec}$ and $60^{\circ} \mathrm{C}$ for $30 \mathrm{sec}$ (40 cycles). Applied Biosystems 7500 software V2.02 (Applied Biosystems; Thermo Fisher Scientific, Inc.) was used with the U6-RNA sequence as an internal control. The $2^{-\Delta \Delta C q}$ method was used to analyze data (12).

Transfection and overexpression of microRNA. The miR-200 mimic and miR-141 mimic were synthesized (Shanghai
Table I. Primer sequences of miR-200 and miR-141.

\begin{tabular}{lc}
\hline Primer & \multicolumn{1}{c}{ Sequence } \\
\hline miR-200-F & 5'-GGTTCTTCCCTGGGCTTC-3' \\
miR-200-R & 5'-GAGTAGGAGCTCCGGATGTG-3' \\
miR-141-F & 5'-CAAACAAAGCCTGGGAGAG-3' \\
miR-141-R & 5'-TTAAGGCCCCAGATTCCAC-3'
\end{tabular}

miR, microRNA; F, forward; R, reverse.

GenePharma Co., Ltd., Shanghai, China) for transfection and overexpression. The medium comprised DMEM with $10 \%$ fetal bovine serum, 5,000 units penicillin and 5,000 units streptomycin. The HCT116 and SW480 colon carcinoma cells (American Type Culture Collection, Manassas, VA, USA) were incubated separately in 96-well plates with cell culture conditions of $37^{\circ} \mathrm{C}$ and $5 \% \mathrm{CO}_{2}$. Following culture for $24 \mathrm{~h}$, lipidosome transfection was performed to establish HCT116 and SW480 cells overexpressing miR-200 or miR-141.

In situ detection of apoptosis using TUNEL staining. The transfected HCT116 and SW480 cells were transferred into 24-well plates following culture for $24 \mathrm{~h}$. Untransfected HCT116 cells were used as a control group, and six parallel experiments were performed for each group. Staining for was performed for the detection of apoptosis at different time points (1, 2, 3 and 4 days) according to the manufacturer's protocol of the apoptosis detection kit (Roche Diagnostics). Subsequently, five clear visual fields were randomly selected under a light microscope to analyze the total cell count and apoptotic cell count. The apoptotic index was calculated as the ratio of apoptotic cells to total cells (13).

Examination of cell migration using a Transwell assay. Matrigel $(60 \mu \mathrm{l} ; 5 \mathrm{mg} / \mathrm{ml})$ was added into the upper Transwell chamber and air-dried at $4^{\circ} \mathrm{C}$. The HCT116 and SW480 cells were cultured, respectively, until the logarithmic growth phase. The cells were digested and resuspended with a diluted cell density of $1 \times 10^{6}$, and $200 \mu \mathrm{l}$ of the cell suspension was added into the upper Transwell chamber. In the lower Transwell chamber, $600 \mu \mathrm{l}$ fresh nutrient medium was added. After $24 \mathrm{~h}$, the Matrigel and HCT116 cells or SW480 cells were cleaned. Crystal violet staining (30 min) was performed for the cells in the lower Transwell chamber. The optical density at $570 \mathrm{~nm}$ was assessed via a microplate assay following rinsing in $10 \%$ acetic acid (14). The above process was repeated three times to produce steady results.

Statistical analysis. SPSS 21.0 software (IBM SPSS, Armonk, NY, USA) was used for data processing. Measurement data are presented as the mean \pm standard deviation. Student's t-test was performed for comparison of difference between two groups. Statistical significance was assessed using one-way analysis of variance among multiple treatment groups. A $\chi^{2}$ test was performed for statistical analysis of enumeration data. $\mathrm{P}<0.05$ was considered to indicate a statistically significant difference. 
Table II. Analysis of the association between clinicopathological parameters and expression levels of miR-200 and miR-141.

\begin{tabular}{|c|c|c|c|c|c|c|}
\hline \multirow[b]{2}{*}{ Parameter } & \multicolumn{3}{|c|}{ Expression of miR-200 } & \multicolumn{3}{|c|}{ Expression of miR-141 } \\
\hline & High (n) & Low (n) & P-value & High (n) & Low (n) & P-value \\
\hline Age (years) & & & $>0.05$ & & & $>0.05$ \\
\hline$\geq 60$ & 118 & 103 & & 121 & 100 & \\
\hline$<60$ & 87 & 72 & & 78 & 81 & \\
\hline Sex & & & $>0.05$ & & & $>0.05$ \\
\hline Male & 122 & 96 & & 115 & 103 & \\
\hline Female & 91 & 71 & & 89 & 73 & \\
\hline Clinical grade & & & $<0.05$ & & & $<0.05$ \\
\hline $\mathrm{I}+\mathrm{II}$ & 46 & 96 & & 37 & 105 & \\
\hline III+IV & 186 & 52 & & 177 & 61 & \\
\hline Liver metastasis & & & $<0.05$ & & & $<0.05$ \\
\hline Yes & 86 & 24 & & 90 & 20 & \\
\hline No & 55 & 215 & & 62 & 208 & \\
\hline $\begin{array}{l}\text { Metastasis in } \\
\text { other organs }\end{array}$ & & & $<0.05$ & & & $<0.05$ \\
\hline Yes & 23 & 9 & & 21 & 11 & \\
\hline No & 66 & 282 & & 60 & 288 & \\
\hline
\end{tabular}

miR, microRNA.
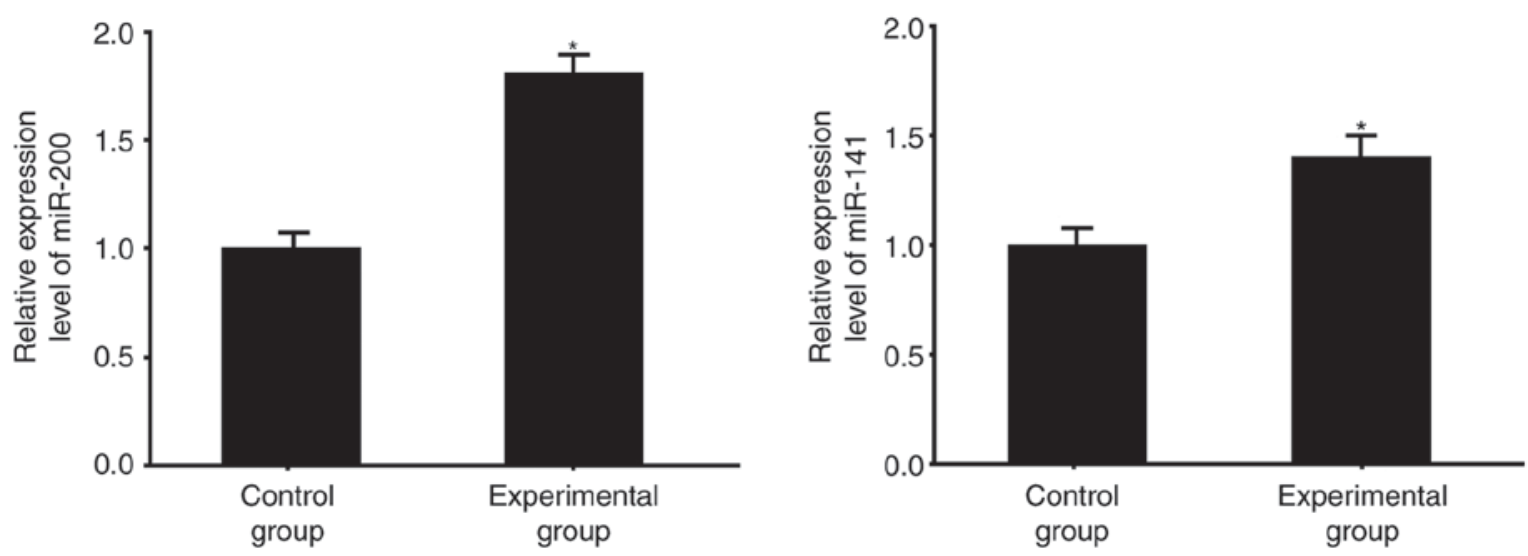

Figure 1. Analysis of mRNA expression levels in the control and experimental groups. (A) mRNA levels of miR-200; (B) mRNA levels of miR-141. ${ }^{*} \mathrm{P}<0.05$, vs. control group. miR, microRNA.

\section{Results}

miR-200 and miR-141 are increased in colorectal cancer. Compared with the normal tissues from the control group, the colorectal cancer tissues had significantly higher mRNA levels of miR-200 and miR-141 ( $\mathrm{P}<0.05$; Fig. 1), which were measured using RT-qPCR analysis.

Increased expression of $\mathrm{miR}-200$ and $\mathrm{miR}-141$ are associated with liver metastasis. The follow-up data showed that 110 patients $(28.9 \%)$ suffered from liver metastasis in the experimental group, whereas 32 patients $(8.4 \%)$ suffered from metastasis in other organs (Table II). Serum levels of miR-200 and miR-141 were positively correlated with clinical grading. In the patients with liver metastasis, $78.2 \%$ had increased expression of miR-200, which was only observed in $20.4 \%$ of the patients without liver metastasis. The same was observed for the expression of miR-141, in that patients with liver metastasis had a higher level, compared with those without liver metastasis (81.8, vs. $22.0 \%$, respectively).

Transfection with miR-200 or miR-141. The overexpression of miR-200 or miR-141 in the HCT116 and SW480 cells was established using mimics. The transfected cells were cultured to the log phase, and total RNA was extracted for RT-qPCR analysis (Fig. 2). Compared with the negative groups, the 
A

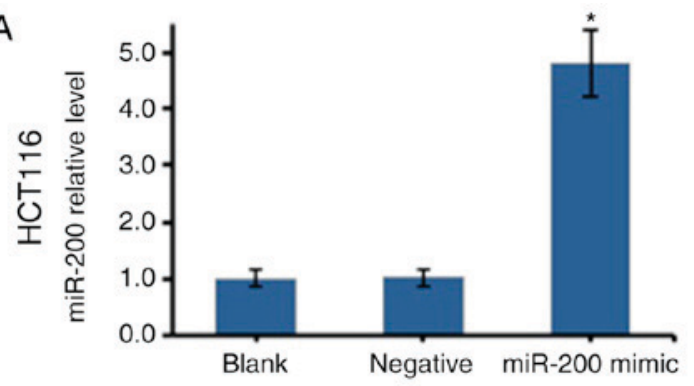

C

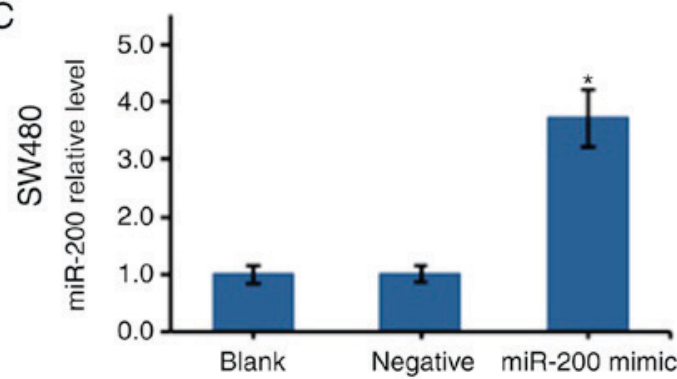

B

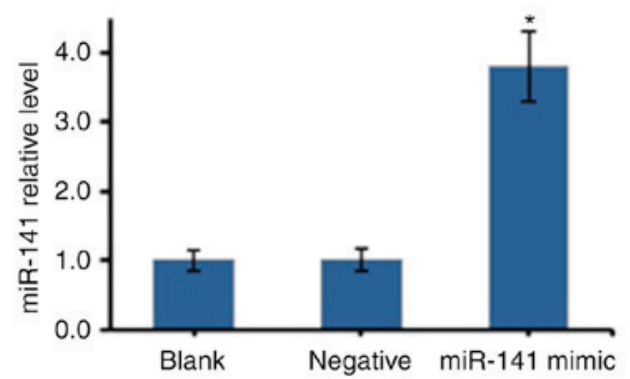

D

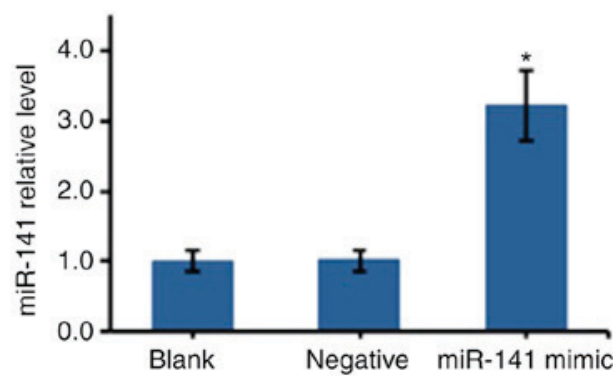

Figure 2. Transfection of cells to overexpress miR-200 or miR-141. Expression of (A) miR-200 and (B) miR-141 in HCT116 cells. Expression of (C) miR-200 and (D) miR-141 in SW480 cells. ${ }^{*} \mathrm{P}<0.05$, vs. negative group. miR, microRNA.
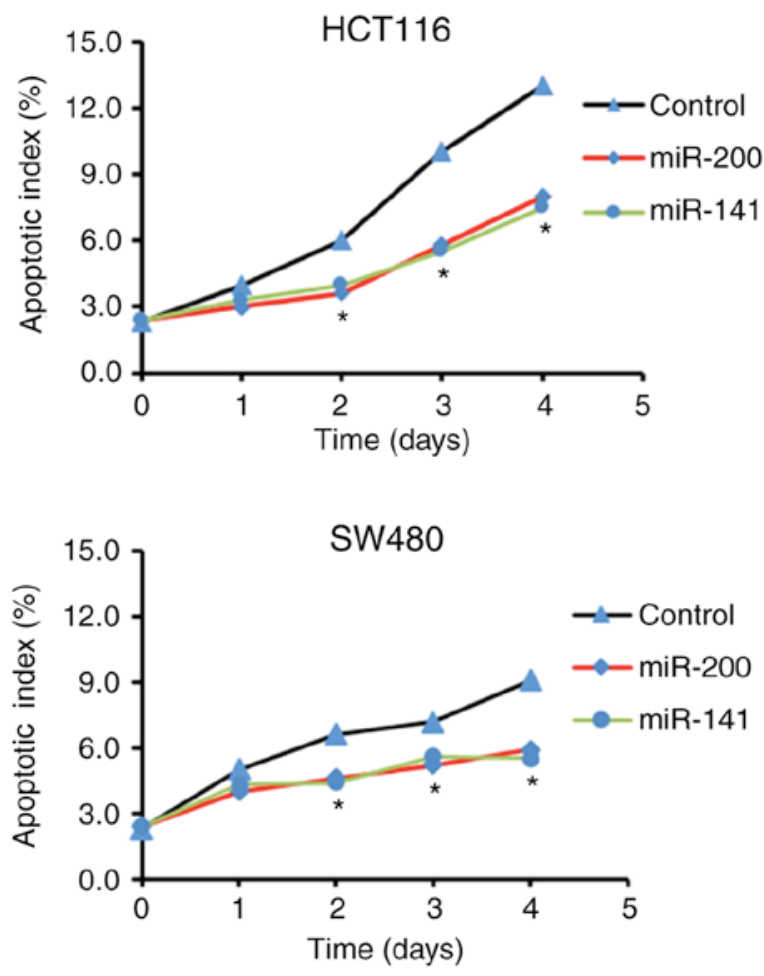

Figure 3. Apoptotic index-time curve. * $\mathrm{P}<0.05$, vs. control group. miR, microRNA.

transfected HCT116 and SW480 cells had significantly higher expression levels of miR-200 and miR-141 ( $\mathrm{P}<0.05)$, suggesting transfection was successful.

miR-200 and miR-141 inhibit the apoptosis of HCT116 and SW480 cells. An apoptosis index-time curve is shown in Fig. 3. Compared with the control groups, the transfected HCT116

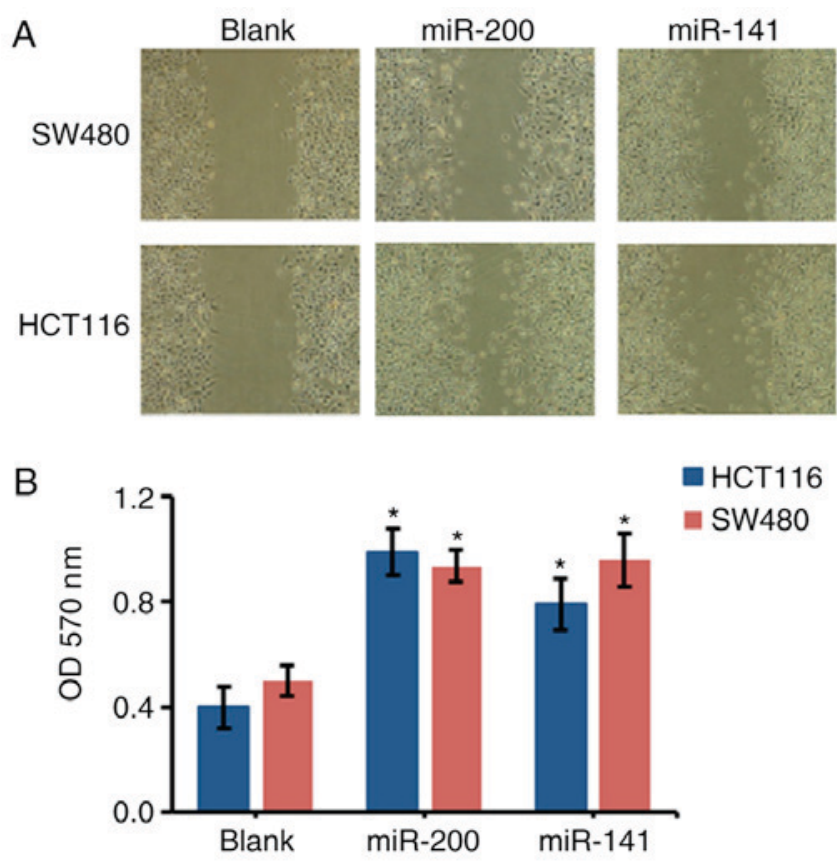

Figure 4. Migration of HCT116 and SW480 cells. (A) Migration in the three groups. (B) Statistical analysis of migration. $\mathrm{P}<0.05$, vs. blank control group. miR, microRNA; OD, optical density.

and SW480 cells had significantly lower apoptotic indices, suggesting that miR-200 and miR-141 inhibited apoptosis.

miR-200 and miR-141 enhance the migration of HCT116 and $S W 480$ cells. Compared with the control groups, the migration of the miR-200-transfected HCT116 and SW480 cells significantly increased. Consistently, the migration of the miR-141-transfected cells was also significantly increased when compared with the blank control group ( $\mathrm{P}<0.05$; Fig. 4). 


\section{Discussion}

Colorectal cancer is the most common type of malignant tumor of the digestive tract in clinical settings, and liver metastasis in colorectal cancer leads to a poorer prognosis (2). Several studies have shown that microRNA is tightly correlated with the development of tumors and survival rates of patients (6). The present study demonstrated that, compared with normal tissues, colorectal cancer tissues expressed significantly higher levels of miR-200 and miR-141. It was also found that miR-200 and miR-141 inhibited apoptosis and enhanced the migration of colorectal cancer cells, suggesting that miR-200 and miR-141 may accelerate liver metastasis in colorectal cancer.

The way in which the profile of microRNAs, including miR-200 and miR-141, is altered in colorectal cancer remains to be fully elucidated (14). Tamagawa et al (15) reported marked demethylation in $\mathrm{CpG}$ regions of the miR-200 and miR-141 promoters in cancer cells; this was confirmed by DNA methylation assays and suggested that cancer cells regulated the profile of microRNAs, particularly miR-200 and miR-141, via DNA methylation. In addition, proteins encoded by microRNA target genes can reversely regulate the profile of microRNAs $(16,17)$. However, further investigations are required to explain how colorectal cancer affects the expression profile of microRNAs.

The migration and invasion of cancer cells are complex biological processes. Studies have shown that epithelialmesenchymal transition (EMT) is pivotal in the migration and invasion of cancer $(18,19)$. Studies have confirmed that miR-200 and miR-141 regulate EMT via affecting the expression of E-cadherin, which is involved in EMT $(20,21)$. When miR-200 and miR-141 are increased, transcription factors of E-cadherin are inhibited, including zinc finger E-box binding homeobox (ZEB)1 and ZEB2, leading to the downregulation of E-cadherin (20). These results are consistent with the findings of the present study.

Early diagnosis and active treatment can improve the survival rates of patients with colorectal cancer. Therefore, it is important to identify effective biomarkers for liver metastasis in colorectal cancer. The present study showed that increased serum levels of miR-200 and miR-141 were associated with liver metastasis in colorectal cancer, and this may improve the accuracy of colorectal cancer and provide a basis for active treatment. In addition, miR-200 and miR-141 enhanced the growth of colorectal cancer, therefore, miR-200 and miR-141 maybe novel therapeutic targets for the treatment of colorectal cancer.

In conclusion, the present study showed that upregulation in the serum levels of miR-200 and miR-141 were associated with liver metastasis in patients with colorectal cancer. The overexpression of miR-200 and miR-141 exacerbated liver metastasis of colorectal cancer via inhibiting apoptosis and inducing migration.

\section{References}

1. Siegel R, Desantis C and Jemal A: Colorectal cancer statistics, 2014. CA Cancer J Clin 64: 104-117, 2014.
2. Abdalla EK, Bauer TW, Chun YS, D'Angelica M, Kooby DA and Jarnagin WR: Locoregional surgical and interventional therapies for advanced colorectal cancer liver metastases: Expert consensus statements. HPB (Oxford) 15: 119-130, 2013.

3. Grothey A, Van Cutsem E, Sobrero A, Siena S, Falcone A, Ychou M, Humblet Y, Bouché O, Mineur L, Barone C, et al: Regorafenib monotherapy for previously treated metastatic colorectal cancer (CORRECT): An international, multicentre, randomised, placebo-controlled, phase 3 trial. Lancet 381: 303-312, 2013.

4. Pritchard CC, Cheng HH and Tewari M: MicroRNA profiling: Approaches and considerations. Nat Rev Genet 13: 358-369, 2012.

5. Friedman RC and Burge CB: MicroRNA target finding by comparative genomics. Methods Mol Biol 1097: 457-476, 2014.

6. Iorio MV and Croce CM: MicroRNA dysregulation in cancer: Diagnostics, monitoring and therapeutics. A comprehensive review. EMBO Mol Med 9: 852, 2017.

7. Schee K, Boye K, Abrahamsen TW, Fodstad $\varnothing$ and Flatmark K: Clinical relevance of microRNA miR-21, miR-31, miR-92a, miR-101, miR-106a and miR-145 in colorectal cancer. BMC Cancer 12: 505, 2012.

8. Faltejskova P, Svoboda M, Srutova K, Mlcochova J, Besse A, Nekvindova J, Radova L, Fabian P, Slaba K, Kiss I, et al: Identification and functional screening of microRNAs highly deregulated in colorectal cancer. J Cell Mol Med 16: 2655-2666, 2012.

9. Zhang L, Yang F, Yuan JH, Yuan SX, Zhou WP, Huo XS, Xu D, Bi HS, Wang F and Sun SH: Epigenetic activation of the MiR-200 family contributes to $\mathrm{H} 19$-mediated metastasis suppression in hepatocellular carcinoma. Carcinogenesis 34: 577-586, 2013.

10. Toiyama Y, Hur K, Tanaka K, Inoue Y, Kusunoki M, Boland CR and Goel A: Serum miR-200c is a novel prognostic and metastasis-predictive biomarker in patients with colorectal cancer. Ann Surg 259: 735-743, 2014.

11. Hamilton SR, Bosman FT and Boffetta P: Carcinoma of the colon and rectum. In: WHO Classification of Tumours of the Digestive System. Bosman FT, Carneiro F, Hruban RH and Theise ND (eds). Lyon, IARC Press, pp134-146, 2010.

12. Kyrylkova K, Kyryachenko S, Leid M and Kioussi C: Detection of apoptosis by TUNEL assay. Methods Mol Biol 887: 41-47, 2012.

13. Stevens P, An Wen Y, Li X and Gao T: Erbin-mediated regulation of colon cancer. FASEB J 29: 724-726, 2015.

14. Ha M and Kim VN: Regulation of microRNA biogenesis. Nat Rev Mol Cell Biol 15: 509-524, 2014.

15. Tamagawa S, Beder LB, Hotomi M, Gunduz M, Yata K, Grenman R and Yamanaka N: Role of miR-200c/miR-141 in the regulation of epithelial-mesenchymal transition and migration in head and neck squamous cell carcinoma. Int J Mol Med 33: 879-886, 2014.

16. Lynch SM, O'Neill KM, McKenna MM, Walsh CP and McKenna DJ: Regulation of miR-200c and miR-141 by methylation in prostate cancer. Prostate 76: 1146-1159, 2016.

17. Gibbons DL, Lin W, Creighton CJ, Rizvi ZH, Gregory PA, Goodall GJ, Thilaganathan N, Du L, Zhang Y, Pertsemlidis A and Kurie JM: Contextual extracellular cues promote tumor cell EMT and metastasis by regulating miR-200 family expression. Genes Dev 23: 2140-2151, 2009.

18. Brabletz T: EMT and MET in metastasis: Where are the cancer stem cells?. Cancer Cell 22: 699-701, 2012.

19. Lu M, Marsters S, Ye X, Luis E, Gonzalez L and Ashkenazi A: E-cadherin couples death receptors to the cytoskeleton to regulate apoptosis. Mol Cell 54: 987-998, 2014.

20. Yu H, Shen Y, Hong J, Xia Q, Zhou F and Liu X: The contribution of TGF- $\beta$ in epithelial-mesenchymal transition (EMT): down-regulation of E-cadherin via snail. Neoplasma 62: 1-15, 2015.

21. Gregory PA, Bert AG, Paterson EL, Barry SC, Tsykin A, Farshid G, Vadas MA, Khew-Goodall Y and Goodall GJ. The miR-200 family and miR-205 regulate epithelial to mesenchymal transition by targeting ZEB1 and SIP1. Nat Cell Biol 10: 593-601, 2008. 